\title{
Doença de Meniere no idoso
}

\author{
Ciríaco C. T. Atherino, ${ }^{1^{*}}$ Aída R. M. de Assunção ${ }^{2}$
}

\section{Resumo}

A doença de Meniere é definida como um conjunto de sintomas que inclui perda auditiva neurossensorial flutuante, vertigem episódica, zumbido e plenitude auricular. Hoje em dia sabe-se que a distensão hidrópica do labirinto é apenas a marca histológica da doença, e não a responsável pelos sintomas. Sua prevalência é baixa na população geral, mas, nas faixas etárias mais avançadas, vem aumentando de frequência, com predomínio do sexo feminino e maior número de comprometimentos bilaterais. O quadro clínico poderia ser descrito como uma sensação inicial de plenitude aural, com surgimento de perda auditiva e zumbido e uma grande crise de vertigem rotatória com náuseas e vômitos. Os exames audiométricos sequenciais firmam o diagnóstico, demonstrando a flutuação da audição. A perda auditiva inicial é geralmente nos sons mais graves, evoluindo progressivamente para o acometimento de todas as frequências. $O$ tratamento pode ser clínico ou cirúrgico. $\mathrm{O}$ tratamento das crises vertiginosas agudas pode ser feito com o paciente internado ou não e deve ser vigoroso. O tratamento da fase crônica é controverso, existindo uma série de medicamentos que podem ser utilizados com resultados discutíveis. Da mesma forma, o tratamento cirúrgico é uma medida de exceção, e visa principalmente acabar com a vertigem, sintoma mais incapacitante da doença.

Descritores: Doença de Meniere; Hidropisia endolinfática; Vertigem.

\section{Abstract \\ Meniere's disease in the elderly}

Meniere's disease is defined as a set of symptoms that include fluctuating hearing loss, episodic vertigo, tinnitus and aural fullness. Nowadays it is known that the hydropic distension of the labyrinth is only the histological hallmark of the disease and is not responsible for the symptoms. Its prevalence in the general population is low, but in the elderly it is increasing in frequency, with female predominance and a higher number of bilateral commitments. The clinical presentation could be described as an initial sense of aural fullness, and the onset of hearing loss and tinnitus and a major crisis of rotational vertigo with nausea and vomiting. Sequential audiometric tests establish the diagnosis, demonstrating a fluctuation in hearing. The initial hearing loss is usually in the low frequencies, and as the time goes by there is involvement of all frequencies. Treatment may be medical or surgical. The treatment of acute vertigo can be done with the patient hospitalized or not and should be vigorous. The treatment of the chronic phase is controversial, and there is a number of medications that can
1. Disciplina de Otorrinolaringologia. Departamento de Especialidades Cirúrgicas. Faculdade de Ciências Médicas. Universidade do Estado do Rio de Janeiro. Rio de Janeiro, RJ, Brasil.

2. Disciplina de Otorrinolaringologia. Departamento de Especialidades Cirúrgicas. Faculdade de Ciências Médicas. Universidade do Estado do Rio de Janeiro. Rio de Janeiro, RJ, Brasil

*Endereço para correspondência:

Serviço de Otorrinolarigologia

Boulevard 28 de Setembro, 77, 5ㅇandar

Rio de Janeiro, RJ, Brasil. CEP: 20551-030.

E-mail: crisatherino@gmail.com

Revista HUPE, Rio de Janeiro, 2015;14(1):66-70

doi: 10.12957/rhupe.2015.17444

Recebido em 28/07/2014. Aprovado em 29/09/2014.

be used with controversial results. Likewise, surgical treatment is a measure of exception, and mainly aims to end dizziness, the most disabling symptom of the disease.

Keywords: Meniere disease; Endolymphatic hydrops; Vertigo.

\section{Resumen}

\section{Enfermedad de Meniere en el adulto mayor}

La enfermedad de Meniere se define como un conjunto de síntomas que incluye la pérdida auditiva neurosensorial fluctuante, vértigo episódico, zumbido y plenitud auricular. Hoy en día se sabe que la distensión hidrópica del laberinto es únicamente la marca histológica de la enfermedad, y no es responsable de los síntomas. Su prevalencia en la población general es baja, pero en los grupos de mayor edad, está aumentado en frecuencia, con predominio del sexo femenino y mayor número de compromisos bilaterales. El cuadro clínico se podría describir como una sensación inicial de plenitud aural, con aparición de pérdida auditiva, zumbido y una gran crisis de vértigo rotatoria con náuseas y vómitos. Los exámenes audiométricos secuenciales afirman el diagnóstico, demostrando la fluctuación en la audición. La pérdida auditiva inicial es generalmente en los sonidos más graves, evolucionando progresivamente hacia la agresión de todas las frecuencias. El tratamiento puede ser clínico o quirúrgico. El tratamiento de las crisis vertiginosas agudas puede ser efectuado con el paciente internado o no y debe ser vigoroso. El tratamiento de la fase crónica es controversial, existiendo una serie de medicamentos que pueden ser 
utilizados con resultados discutibles. Asimismo, el tratamiento quirúrgico es una medida excepcional, y tiene como objetivo principalmente acabar con el vértigo, el síntoma más

\section{Introdução}

A doença de Meniere é definida como sendo um conjunto de sintomas que incluem perda auditiva neurossensorial flutuante, vertigem episódica, zumbido e plenitude auricular. ${ }^{1}$

Foi primeiramente descrita na França, em 1861, por Prosper Meniere, eminente médico francês. Na ocasião, este apresentou um paciente portador de vertigem, perda auditiva e zumbido, sintomas que ele atribuiu a uma disfunção do ouvido.

A partir desta data muito se estudou sobre este assunto e, até hoje, muita controvérsia existe a partir até mesmo do nome: síndrome ou doença de Meniere? Se consultarmos o dicionário Aurélio, o verbete "síndrome" diz respeito a "estado mórbido caracterizado por um conjunto de sinais e sintomas e que pode ser produzido por mais de uma causa, como por exemplo: síndrome de obstrução intestinal,...". O que se verificou no tempo foi que esta tríade, vertigem episódica, perda auditiva flutuante, zumbido, e mais plenitude auricular, poderia ocorrer numa série de disfunções labirínticas, a saber: genéticas, infecciosas, vasculares, nutricionais, alérgicas, autonômicas, endócrinas e autoimunes. ${ }^{1}$ Portanto, a síndrome de Meniere se apresentava nestas doenças. Entretanto, quando não se conseguia verificar uma causa para o aparecimento desta sintomatologia, caso em que, aí sim, teríamos a "doença de Meniere", a presença da síndrome de natureza idiopática.

Mais tarde, em 1938, na Inglaterra, Hugh Cairns e Charles Hallpike atribuíram a existência de uma hidropisia endolinfática como sendo a causa histopatológica da síndrome de Meniere. Mas, hoje em dia, após o trabalho de Merchant S e colaboradores, de 2006, ${ }^{2}$ em que foram analisados 1750 ossos temporais de 963 pacientes, verificou-se que a hidropisia endolinfática deve ser considerada muito mais a marca histológica da síndrome de Meniere do que a responsável direta por seus sintomas.

Quanto à epidemiologia, a síndrome acomete especialmente a faixa entre 40 e 50 anos, é excepcional em crianças e existem fatores genéticos predisponentes. ${ }^{1}$

Quanto à prevalência, esta fica em torno de 0,12\% na população geral e 1,6\% com relação às vertigens de origem vestibular. ${ }^{3}$ incapacitante de la enfermedad.

Palabras clave: Enfermedad de Meniere; hidropesía endolinfática; vértigo.

E no idoso? Vários trabalhos têm demonstrado que: (1) está aumentando o número de casos com início dos sintomas acima dos 60 anos de idade, (2) está aumentando o número de mulheres acometidas, e (3) está também aumentando o número de casos bilaterais nesta faixa etária.4-6

Van Leeuwen RB e colaboradores $(2014)^{5}$ analisaram 731 pacientes acima de 70 anos e verificaram que a síndrome de Meniere atingia 5,2\% deles e que as mulheres surgiam numa proporção de quase 2 para 1.

Outros trabalhos, como o de Uneri A. e colaboradores (2008), ${ }^{6}$ com 601 pacientes acima de 65 anos, encontrou percentual ainda maior: $12,47 \%$.

Mas é bom frisar que a causa mais comum de tonteira mesmo em pacientes acima de 70 anos continua a ser a vertigem postural paroxística benigna que, em algumas estatísticas, atinge $53,3 \%$ dos casos de vertigem nos idosos.

A fisiopatologia da hidropisia endolinfática é desconhecida, existindo várias teorias: obstrução do duto / saco endolinfático, alterações na absorção da endolinfa, alterações na produção da endolinfa, agressão autoimune, origem vascular, etiologia viral, entre outras. Em suma, todas elas provocariam problemas ou na produção ou na reabsorção da endolinfa (ou em ambas as etapas), propiciando seu acúmulo no labirinto membranoso, e daí a distensão hidrópica. ${ }^{1}$

O quadro clínico poderia ser descrito como uma sensação inicial de plenitude aural, com surgimento de perda auditiva e zumbido e uma grande crise de vertigem rotatória com náuseas e vômitos. Esta última é realmente o problema mais impactante e que leva o paciente ao médico. ${ }^{1}$ Esta sintomatologia pode se apresentar de forma completa ou não. Normalmente, a crise vertiginosa é desencadeada por estresse, fadiga e mesmo variações da pressão atmosférica. É acompanhada, geralmente, de sinais neurovegetativos importantes, sua duração é de 2 a 3 horas e a frequência é variável. Como as crises são imprevisíveis, ocorrem o medo e a insegurança. É interessante saber que o paciente, na maioria dos casos, apresenta um perfil psicológico particular: inteligente, com grande cultura, meticuloso, perfeccionista beirando a obsessão Muitas vezes a cefaleia acompanha este quadro. Alguns autores associam a surdez súbita nos sons graves com o desen- 
volvimento posterior de doença de Meniere. Junicho M. e colaboradores $(2008)^{8}$ seguiram 184 pacientes que apresentaram este quadro súbito e verificou que 17 deles (9\%) desenvolveram a doença.

Quanto à bilateralização, existe uma grande variação de informes na literatura. ${ }^{1}$ A maioria mostra que, após 2 anos de evolução, 15\% dos casos se tornam bilaterais, e, após 10 a 20 anos de evolução, 30\% a 60\%.

Os exames audiométricos sequenciais são indispensáveis para o diagnóstico. ${ }^{1}$ No início da doença podemos encontrar uma perda auditiva maior nos sons mais graves (Figura 1), com boa discriminação verbal.

Na evolução, verificamos alguns audiogramas que podem demonstrar a flutuação da audição, mas sempre com uma evolução descendente, até que há uma estabilização (Figura 2) com comprometimento tardio de todas as frequências em torno de $50 \mathrm{~dB}$ a $70 \mathrm{~dB}$.

O paciente pode apresentar hiperacusia (acuidade auditiva exacerbada) e/ou diplacusia (escuta do mesmo som de forma diferente pelos 2 ouvidos). Raramente ocorre cofose, ou seja, a perda completa da audição. A timpanometria é normal, do tipo A (Jerger), o que era esperado, já que não há comprometimento da Orelha Média. A pesquisa dos reflexos estapédicos pode revelar um recrutamento de Metz, ou seja, os limiares dos reflexos e os limiares auditivos estão mais próximos que o normal (diferença por frequência menor que 60dB). A presença de recrutamento indica compressão do campo

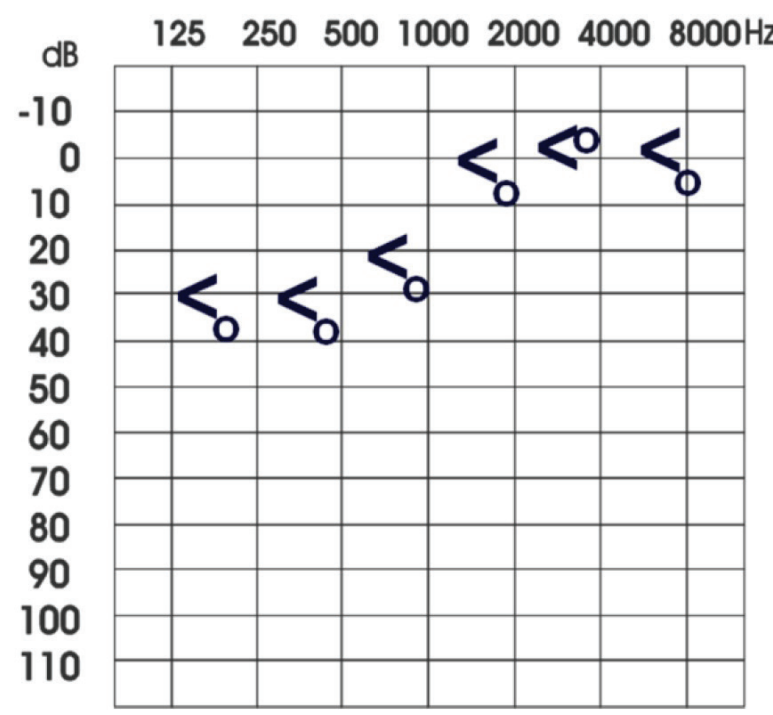

Figura 1. Audiograma mostrando apenas a marcação do ouvido direito. Observa-se perda auditiva moderada em sons graves, característica muitas vezes do início da doença. auditivo, ou seja, o limiar de audibilidade está muito próximo do limiar de irritabilidade, característica das lesões de origem coclear, na Orelha Interna.
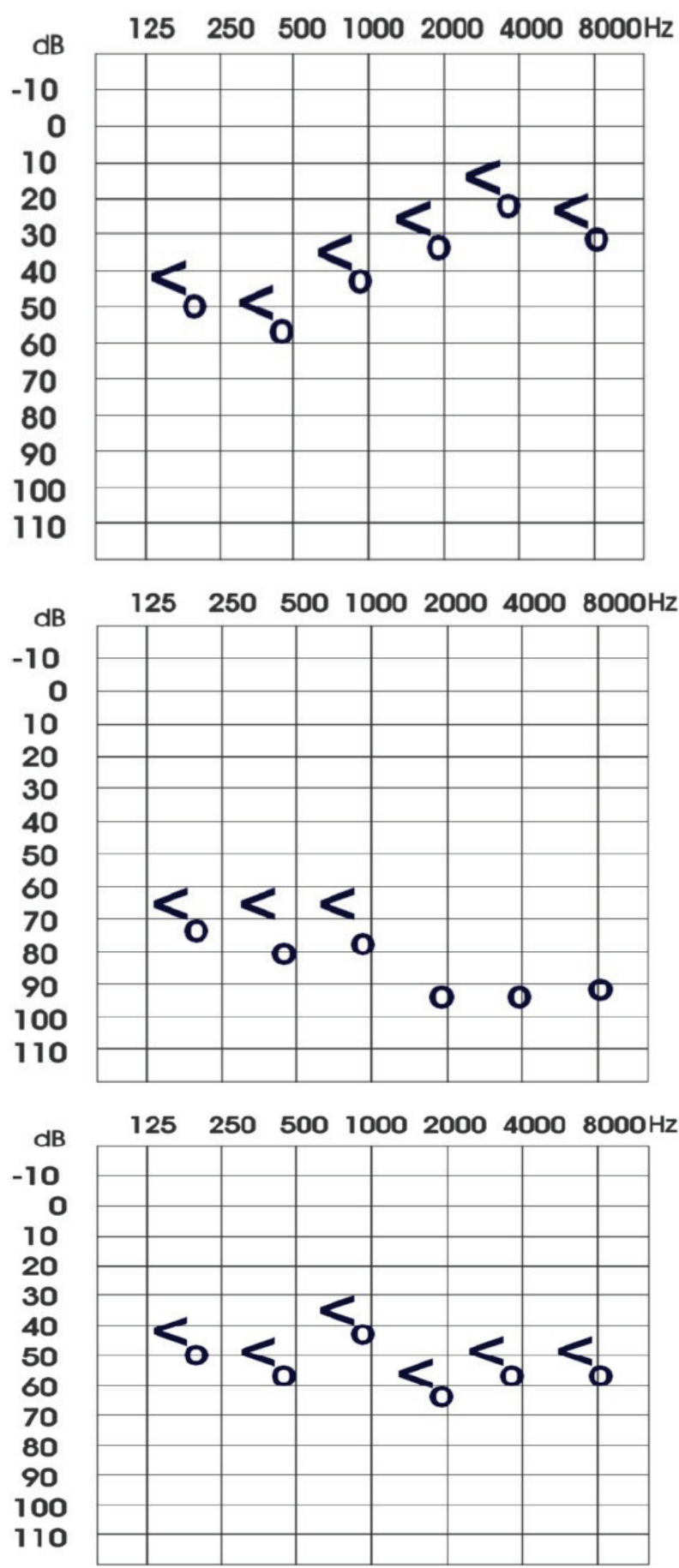

Figura 2. Evolução audiométrica da doença de Meniere. Observe-se a flutuação da audição no ouvido direito nos diferentes audiogramas.. 
Outro teste também utilizado é a eletrococleografia, na qual se procura a relação entre o potencial de somação (SP - summating potential) e o potencial de ação (AP - action potential). Esta relação é considerada normal até $36 \%$. Acima disto pode-se suspeitar de hidropisia endolinfática.

Os potenciais vestibulares miogênicos evocados (VEMP) estão cada vez mais sendo utilizados para diagnóstico precoce da doença. Estes são reflexos desencadeados por estímulo acústico do sáculo causando inibição da atividade do músculo esternocleidomastoídeo ipsilateral. Portanto, o exame testa a função do sáculo e do nervo vestibular inferior. Verificou-se que o limiar dos mesmos está aumentado utilizando-se as frequências de $500 \mathrm{~Hz}$ e $250 \mathrm{~Hz}$ em $80 \%$ dos casos de Meniere. $^{9}$

Quanto ao tratamento, este pode ser clínico ou cirúrgico. Os objetivos do mesmo são:

1. Tratar a crise.

2. Prevenir o advento de outras crises.

3. Melhorar e/ou preservar as funções coclear e vestibular.

4. Evitar o desenvolvimento de uma doença bilateral.

\section{Tratamento clínico}

\section{Tratamento da crise}

1. Repouso.

2. Hospitalização (se necessário).

3. Antivertiginosos.

4. Antieméticos.

5. Sedativos.

6. Manitol, neurolépticos, corticoides etc... (se necessário).

A crise vertiginosa é um episódio dramático e deve ser tratado com presteza. Nossa preferência é a associação dimenidrinato + piridoxina por via intramuscular ou endovenosa a cada 8 ou 12 horas. A isto pode-se associar o diazepam 10mg injetável. Bromoprida, metoclopramida e ondansetrona são recursos adicionais que podem ser também utilizados principalmente no controle das náuseas e vômitos. Também os corticosteroides podem ser usados, já que podem reduzir a duração da crise vertiginosa e também potencializar o efeito antiemético da ondansetrona. Nossa preferência é a dexametasona $4 \mathrm{mg}$ intramuscular ou endovenosa. Cabe lembrar que não se deve utilizar soro glicosado na manutenção da via venosa, pois o mesmo é um irritante labiríntico. ${ }^{10}$
Logo que a crise aguda seja controlada é interessante reduzir e retirar a medicação já que há evidências de que a mesma pode retardar o processo de compensação vestibular.

Quanto à fase crônica, James AL e colaboradores $(2005)^{11}$ analisou o papel da betahistina na síndrome de Meniere, vasodilatador que diminuiria a isquemia na orelha interna permitindo melhor metabolismo da endolinfa, e não encontrou evidências convincentes para seu uso. Entretanto, muitos autores utilizam esta substância na fase crônica da doença, alguns até em dosagens superiores às recomendadas pelos laboratórios, com bons resultados relatados.

Quanto aos diuréticos, Thirlwall AS e colaboradores (2006) $)^{12}$ também não encontrou evidências suficientes para seu uso, embora alguns pacientes refiram melhoria.

\section{Tratamento cirúrgico}

A indicação principal do tratamento cirúrgico diz respeito às vertigens incapacitantes que não respondem ao tratamento clínico e quando já ocorre sensível deterioração da função auditiva associada. Define-se síndrome/doença de Meniere incapacitante como a ocorrência de mais de quatro episódios de vertigem por mês por, pelo menos, 3 meses.

A primeira opção hoje em dia é a labirintectomia química, cuja indicação é eliminar crises recorrentes de vertigem em síndrome/doença de Meniere unilateral. Utiliza-se a gentamicina intratimpânica, tendo como princípio do tratamento o fato de que as células ciliadas vestibulares são mais sensíveis à gentamicina que as cocleares. A filosofia do tratamento é abolir a função vestibular preservando parte da função para evitar sequelas equilibratórias.

Quando isto não dá certo é possível realizar uma descompressão do saco endolinfático, cirurgia que visa liberar esta estrutura de forma a descomprimir todo o labirinto membranoso. Seus resultados são também discutíveis. Ainda como opções cirúrgicas temos a cocleosaculotomia (labirintectomia setorial mais reservada para os idosos), as labirintectomias em geral (transcanal e transmastoídea) e, como solução final, a neurectomia vestibular.

\section{Conclusão}

Em suma, apesar de alguns avanços no diagnóstico e tratamento nestes anos recentes, a doença de Meniere ainda constitui um desafio. Sua fisiopatologia ainda não foi completamente elucidada e, até que sejam feitos 


\section{Artigo de revisão}

avanços nesta área, éimprovável que sejam descobertos tratamentos mais efetivos. ${ }^{1}$

\section{Referências}

1. Kirtane MV, Souza CE. Otology and Neurotology. Delhi, Thieme Medical and Scientific Publishers, 2013.

2. Merchant SN, Adams JC, Nadol Jr JB. Pathophysiology of Ménière's Syndrome: Are Symptoms Caused by Endolymphatic Hydrops? Otology \& Neurotology 2005;26:74-81.

3. Radtke A, von Brevern M, Feldmann M, Lezius F, Ziese T, Lempert T, et al. Screening for Meniere's disease in the General Population. The Needle in the Haystack. Acta Otolaryngol. 2008;128(3):272-276.

4. Shojaku H, Watanabe Y, Yagi T, Takahashi M, Takeda T, Ikezono $\mathrm{T}$, et al. Changes in the characteristics of definite Meniere's disease over time in Japan: a long-term survey by the Peripheral Vestibular. Disorder Research Committee of Japan, formerly the Meniere's Disease Research Committee of Japan. Acta Otolaryngol. 2009;129(2):155-60. http://dx.doi. org/10.1080/00016480802112587

5. van Leeuwen RB, Bruintjes TD. Dizziness in the elderly: Diag- nosing its causes in a multidisciplinary dizziness unit. Ear Nose Throat J. 2014;93(4-5):162,164,166-7.

6. Uneri A, Polat $\mathrm{S}$. Vertigo, dizziness and imbalance in the elderly. J Laryngol Otol. 2008;122(5):466-9.

7. Lüsher M, Theilgaard S, Edholm B. Prevalence and characteristics of diagnostic groups amongst 1034 patients seen in ENT practices for dizziness. J Laryngol Otol. 2014;128(2):128-33. http://dx.doi.org/10.1017/S0022215114000188

8. Junicho M, Aso S, Fujisaka M, Watanabe Y. Prognosis of Low-Tone Sudden Deafness. Does It Inevitably Progress to Meniere's disease? Acta Otolaryngol. 2008;128(3):304-8. http:// dx.doi.org/10.1080/00016480601002096

9. Rauch SD, Silveira MB, Zhou G, Kujawa SG, Wall C 3rd, Guinan JJ. Vestibular evoked myogenic potentials versus vestibular test battery in patients with Meniere's disease. Otol Neurotol. 2004 Nov;25(6):981-6.

10. Atherino CCT. Terapêutica Farmacológica da Vertigem. Revista HUPE. 2012;11(4):36-41.

11. James AL et alii. Betahistine for Meniere's disease or syndrome. Cochrane Database of Syst Rev. 2005;2.

12. Thirlwall AS, Kundu S. Diuretics for the treatment of Ménière's disease or syndrome. Cochrane Database Syst Rev. 2006:3. 